\title{
TOWARDS A BIOMECHANICAL MODEL OF THE LARYNX
}

\author{
Arthur Lobo and Michael O'Malley \\ BerkeleySpeechTechnologies \\ 2246 Sixth St. \\ Berkeley, CA 94710 \\ U.S.A.
}

\begin{abstract}
In this paper, the development of a large-displacement large-strain 3-D finite element model of the vocal fold is reported. A fold is discretized into 720 8-node brick elements with a total of 1001 nodes and 3003 displacement degrees of freedom. The structure has realistic dimensions and geometry. The model includes geometric and material nonlinearities. The geometric nonlinearity appears in the strain-displacement relation due to the second order displacement derivatives and the material nonlinearity refers to the constitutive law. The Mooney-Rivlin rubber material formulation for an anisotropic tissue medium is used to characterize the tissue rheology. The elasticity tensor and the stress tensor for the Total Lagrangian formulation are obtained from the partial derivatives of the strain energy density function (SEDF) with respect to the Green-Lagrange strain tensor. Incompressibility constraints have been added using a mixed displacement-pressure (1 constant pressure term) finite element - a hydrostatic pressure work term (Lagrange Multiplier) being added to the SEDF. The structure is subjected to a sinusoidally time-varying half cosine pressure profile applied on 117 medial surface nodes. The dynamic equilibrium equations are solved using an incremental-iterative strategy and the Newmark method of time integration for the implicit initial-boundary-value problem. The deformation of the vocal fold at various phases of the applied load was studied.
\end{abstract}

\section{INTRODUCTION}

The vocal cords are responsible for the quasi-periodic excitation of the vocal tract during voiced speech production. At present not much is known about the detailed movements of the different regions of the vocal fold tissue during a cycle of vibration. Such knowledge and simulation capability is of interest to otolaryngologists in enabling them to predict vocal outcome before surgical manipulation of structurally pathological vocal cords, as seen for eg., in cases of dysphonia due to polyp, amyloid tumor, sulcus vocalis, etc. A complete model of the larynx would be invaluable in providing insight into how best to perform laryngeal framework surgery, for eg., thyroplasty, in cases of blunt larynx trauma. In the next section, the finite element implementation of the vocal fold is discussed. The following section deals with simulation of a vibrating vocal fold. The concluding section discusses directions for further work.

\section{IMPLEMENTATION}

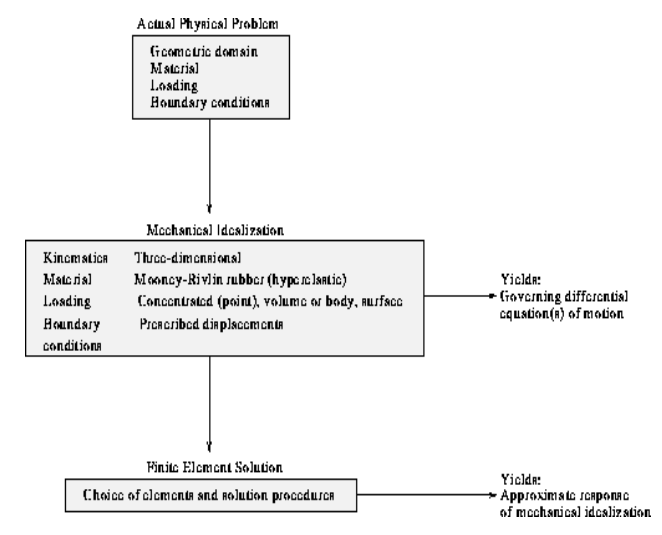

Figure 1: Finite element solution process.

Initially, a three-dimensional static linear finite element model of the vocal fold was developed. The finite element solution process is illustrated in Fig. 1. The geometry and dimensions of the vocal fold continuum were adapted from Titze and Talkin (1979). The structure was discretized into $720(6 \times 10 \times 12) 8$-node isoparametric hexahedron (trilinear) elements with 6 elements in the $\mathrm{x}$ direction medial surface to thyroid cartilage, 10 elements in the $\mathrm{y}$ direction - vocal processes to anterior commissure, 
and 12 elements in the $\mathrm{z}$ direction - superior surface to inferior surface (see Fig. 3). This corresponded to 1001 (7x11x13) displacement nodes and 3003 (3x1001) displacement degrees of freedom. Hirano's anatomical studies (1977) of the larynx along with the mechanical properties of the various layers provides a rationale for the layered structure implementation of the vocal fold as discussed next. A schematic presentation of the structure of the human vocal fold adapted from Hirano (1977) is shown in Fig. 2 for comparison.

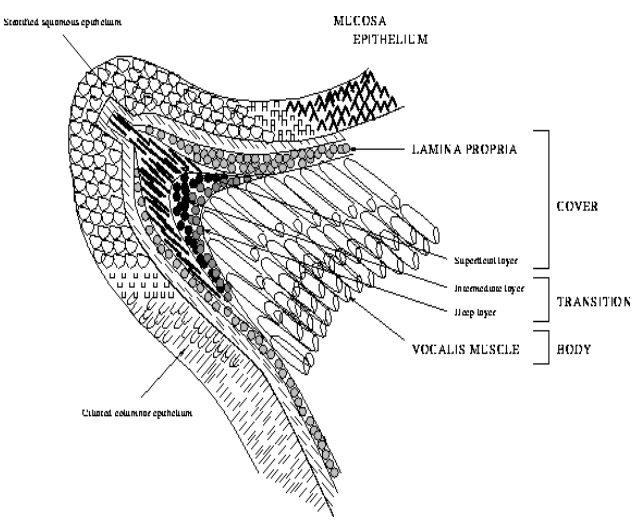

Figure 2: Schematic presentation of a transverse section of the human vocal fold.

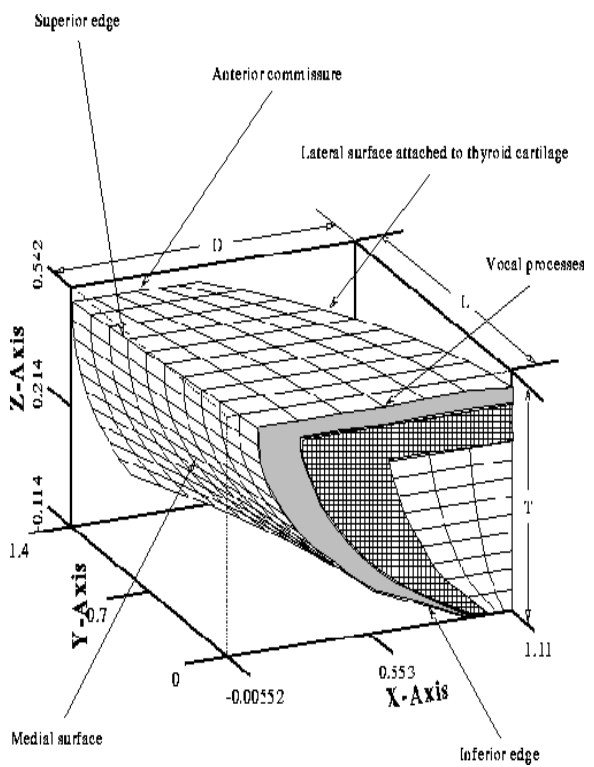

Figure 3: Finite element discretization of the vocal fold.
Three sets of orthotropic (Lai, et al., 1993) material properties for the elements were defined, one for each layer. An element was of the mucosa (cover) type if it was in the outermost element layer of the medial and superior surfaces $(0.05 \mathrm{~cm}$ thickness), the ligament type if it was in the next two element thickness layer from the medial and superior surfaces $(0.1 \mathrm{~cm})$, and the Thyroarytenoid muscle type if it was in the remainder of the structure. In Fig. 3, the lightly shaded elements represent mucosal tissue, the darker elements represent the ligament and the clear elements represent the thyroarytenoid muscle tissue. $\mathrm{L}$ is the length, $\mathrm{D}$, the depth and $\mathrm{T}$, the thickness. All nodes on the anterior commissure, vocal processes and lateral surface are fixed for the essential boundary conditions. The dynamic case was simulated by adding mass (for inertia) and damping (for viscosity) matrices and introducing nodal accelerations and velocities as state variables in addition to the nodal displacements from the static formulation. The load was a time varying pressure profile on 117 medial surface nodes as discussed in the section on Simulation.

The finite element model was extended to include material and geometric nonlinearities, and tissue incompressibility. A hybrid displacement(u)-pressure(p) finite element was used, namely the $8 \mathrm{u} / 1 \mathrm{p}$ element. The discontinuous pressure term was statically condensed out at the element level so that the bandwidth of the stiffness matrix remained the same. An incremental-iterative Total Lagrangian formulation (Sussman and Bathe, 1987) was implemented.

\subsection{Constitutive Relation}

Vocal fold tissue can be considered as a hyperelastic rubber-like material characterized by a strain energy density function (SEDF). Constitutive (stress-strain law) modeling schemes based on SEDF were introduced to soft tissue biomechanics by Fung and are extensively summarized in a text by Fung (1981). For the present work, the nearly incompressible Mooney-Rivlin rubber material formulation (Oden, 1972) was used to obtain the tangent constitutive matrix and the stress tensor. The tissue medium was assumed to be anisotropic. The evaluation of the components of the stress tensor and the constitutive tensor is described in tensor notation in Sussman and Bathe (1987), and in matrix notation in Gadala(1992). 


\section{SIMULATION}

The force was restricted to the $\mathrm{x}$ direction. The equation for the pressure profile was a half cosine shape sinusoidally modulated in time,

$\mathrm{Fx}=\mathrm{F} \sin (2$ pi f n DEL t $) \cos \left(\mathrm{pi} / \mathrm{T} \mid \mathrm{Z} \_\right.$node sup $\mathrm{Y}_{-} \mathrm{i}-$ Z_lat sup $Y_{-}$i|)

where $\mathrm{F}$ is the amplitude of the applied force in dynes, $\mathrm{f}$ is the frequency of vibration in $\mathrm{Hz}, \mathrm{n}$ is the $\mathrm{nth}$ time step of size DEL $t, T$ is the thickness of the fold at the vocal processes $(Y=0), Z$ node sup $Y \_i$ is the $Z$ coordinate of the medial surface node at the ith $Y$-plane and $Z_{-}$lat sup $\mathrm{Y} \_\mathrm{i}$ is the $\mathrm{Z}$ coordinate of the bottom-most node on the lateral surface at the ith Y-plane. The argument of the cosine function is therefore the vocal fold thickness at the ith Y-plane normalized by the thickness at the vocal processes. Fx is calculated for all 117 nodes on the medial surface. The Newmark method of time integration for the implicit initial-boundary-value problem was used (Bathe et al., 1975).

For the simulation, the parameter values were: $\mathrm{F}=4.0$ dynes, $\mathrm{f}=100 \mathrm{~Hz}$, DEL $\mathrm{t}=0.0001 \mathrm{sec}$, length $(\mathrm{L})=1.4$ $\mathrm{cm}$, thickness at vocal processes $(\mathrm{T})=0.5 \mathrm{~cm}$, depth at vocal processes $(D)=1.0 \mathrm{~cm}$, depth at anterior commissure $=0.5 \mathrm{~cm}$. For the material law, the Mooney-Rivlin constants, C_1 $=1813.4$ dynes per sq. cm., C_ $2=259.1$ dynes per sq. cm., bulk modulus $=166,666.7$ dynes per sq. cm. Figures 4-9 show the vocal fold deformation at different phases (between 0 and 4 pi or 2 cycles) of the applied external pressure profile.

\section{SUMMARY}

This project is aimed at developing a biomechanical model of the vocal folds. One aspect of the work involves the use of the finite element method to model the structural and material properties of the folds and their dynamics in normal and pathological (cord structure-related) voices. The following outlines areas where further work will be done: Anatomical information, specifically layering and fibre directions from a real larynx will be integrated into the finite element model. The vocal fold contact-impact constraints will be imposed using an Augmented Lagrangian Multiplier method with a suitable frictional criterion. A biphasic constitutive law will be implemented to describe the hydrated tissue. Dynamic nonlinear viscoelasticity under transient loading conditions such as at glottal opening and closure (small time scale), voice onset or rapid pitch change (large time scale), will be modeled by using relaxation functions for describing the time-variation of the Mooney-Rivlin rubber material coefficients. A hybrid finite element with a higher interpolation order (than the element in use) for the displacement (u) and pressure (p) variables, namely the $27 \mathrm{u} / 4 \mathrm{p}$ element will be used. The glottal aerodynamic forces will be simulated with a model of fluid flow in the vocal tract and used to drive the vocal fold model. Finally, it is proposed to generate the active stress component as a function of electromyogram data recorded from the Thyroarytenoid muscle.

\section{REFERENCES}

[1] Bathe, K. J., Ramm, E., and Wilson, E. L. "Finite element formulations for large deformation dynamic analysis," Int. J. Num. Meth. Eng. 9, 353-386, 1975.

[2] Fung, Y. C. 1981. Biomechanics, Mechanical Properties of Living Tissues, Springer-Verlag, New York, 1981.

[3] Gadala, M. S. "Alternative methods for the solution of hyperelastic problems with incompressibility," Computers and Structures, 43(1), 1-10, 1992.

[4] Hirano, M. "Structure and vibratory behaviour of the vocal folds," in Dynamic Aspects of Speech Production - Current Results, Emerging Problems, and New Instrumentation, edited by M. Sawashima and F. S. Cooper, University of Tokyo,Japan, 1977.

[5] Lai, W. M., Rubin, D., and Krempl, E. Introduction to Continuum Mechanics, Pergamon, New York, 1993.

[6] Oden, J. T. Finite Elements of Nonlinear Continua, McGraw-Hill, New York, 1972.

[7] Sussman, T., and Bathe, K. J. "A finite element formulation for nonlinear incompressible elastic and inelastic analysis," Computers and Structures, 26(1/2), 357-409, 1987.

[8] Titze, I. R., and Talkin, D. "A theoretical study of the effects of various laryngeal configurations on the acoustics of phonation," J. Acoust. Soc. Am., 66(1), 60-74, 1979. 


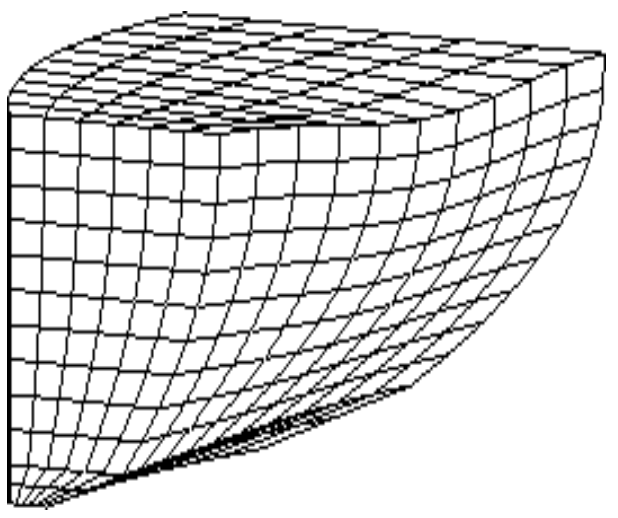

Figure 4: Vocal fold deformation at phase pi of pressure profile on medial surface.

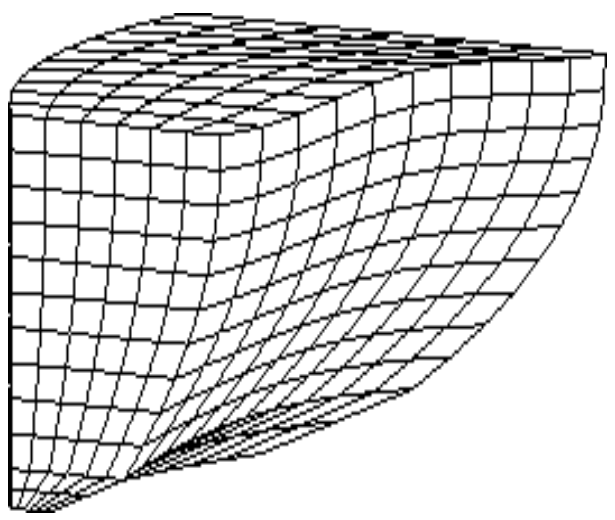

Figure 5: Vocal fold deformation at phase $11 \mathrm{pi} / 5$ of pressure profile on medial surface.

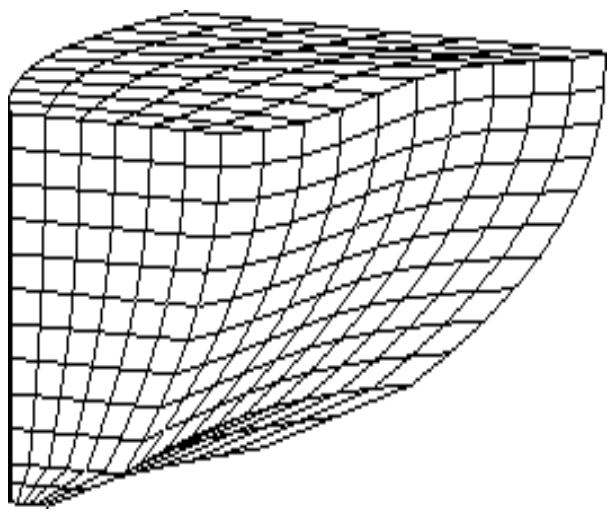

Figure 6: Vocal fold deformation at phase $12 \mathrm{pi} / 5$ of pressure profile on medial surface.

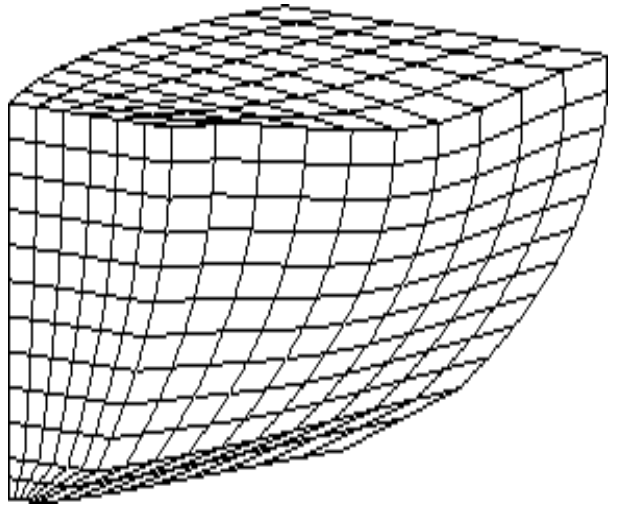

Figure 7: Vocal fold deformation at phase 3 pi of pressure profile on medial surface.

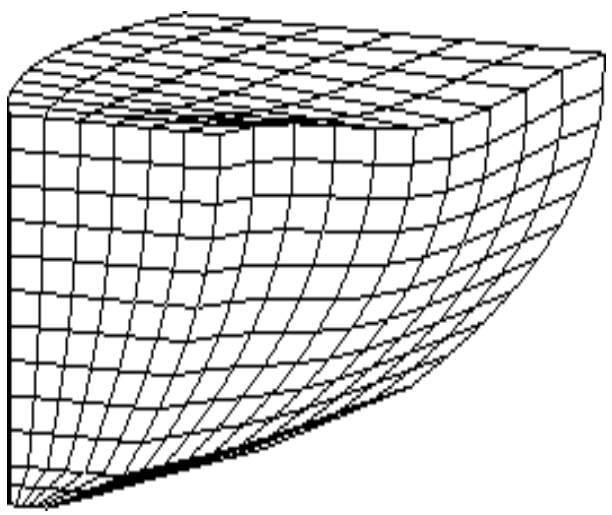

Figure 8: Vocal fold deformation at phase $16 \mathrm{pi} / 5$ of pressure profile on medial surface.

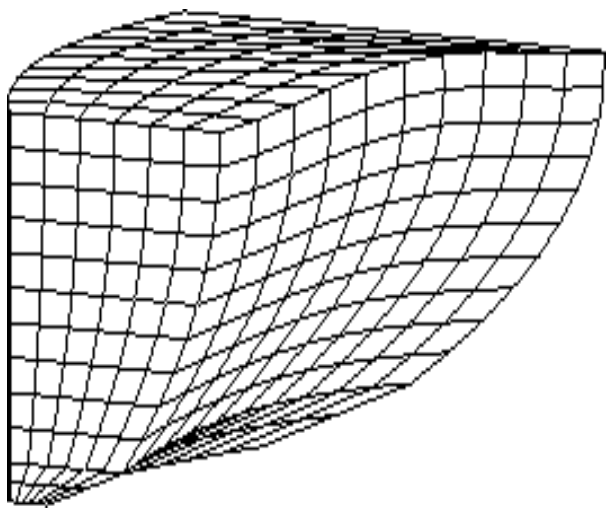

Figure 9: Vocal fold deformation at phase 4 pi of pressure profile on medial surface. 